\title{
GESSO NA PRODUÇÃO DE CULTIVARES DE MILHO COM TOLERÂNCIA DIFERENCIAL A ALUMíNIO EM TRÊS NÍVEIS DE CALAGEM(1)
}

\author{
B. van RAIJ (2), P. R. FURLANI (2), J . A. \\ QUAGGIO(2) \& A. PETTINELLI J ÚNIOR ${ }^{(3)}$
}

\begin{abstract}
RESUMO
O gesso, nos últimos anos, vem sendo considerado como um insumo capaz de melhorar o ambiente radicular de subsolos ácidos. Contudo, ainda pairam dúvidas sobre a vantagem de usá-lo em solos que, embora ácidos, tenham sido submetidos a calagens e adubações anteriores. Também há pouca informação sobre o uso de gesso em presença de aplicações elevadas de calcário ou em plantas cultivadas tolerantes à acidez. No presente trabalho, é relatado experimento com calcário e gesso, realizado, de 1987 a 1992, na Estação Experimental de Tatuí (SP), em Latossolo Vermel ho-E scuro álico textura argi losa, com o objeti vo de avaliar o efeito de calcário e de gesso nas produções de cultivares de mi l ho tolerante ou susceptível a alumínio, bem como o efeito dos corretivos na acidez do solo. 0 experimento foi instalado em parcelas subsubdivididas, com quatro repetições, em blocos ao acaso. Nas parcelas princi pais, foram aplicadas 0,6 ou $12 \mathrm{t} \mathrm{ha}^{-1}$ de calcário dolomítico e, nas subparcelas, 0,4 e $8 \mathrm{t} \mathrm{ha}^{-1}$ de fosfogesso; nas subsubparcelas, foram plantados dois cultivares, um sensível e outro tolerante a alumínio. Para as quatro colheitas obtidas (os dados de 1990 foram considerados perdidos), percebeu-se efeito significativo para a calagem nos dois tipos de cultivares. $O$ gesso apresentou efeito significativo nas produções apenas para o cultivar sensível ao alumínio e, nesse caso, o efeito foi aditivo ao de calcário, proporcionando, em média, cerca da metade do aumento de produção devida à calagem. A calagem influenciou, consideravel mente, a reação da camada arável do solo, aumentando o $\mathrm{pH}$, os teores de $\mathrm{Ca}^{2+} \mathbf{e} \mathrm{Mg}^{2+}$ e reduzindo a acidez potencial, mas pouco influiu nas camadas mais profundas, o que, provavelmente, se deveu ao fato de o solo ter recebido aplicações anteriores de calcário e de adubos com sulfato. $\mathrm{O}$ gesso influiu nos teores de $\mathrm{Ca}^{2+}$ e $\mathrm{SO}_{4}{ }^{2-} \mathrm{em}$ profundidade, embora de forma pouco pronunciada em relação às quantidades aplicadas, e não alterou as características de acidez. Pode-se concluir que, mesmo tendo alterado pouco as características químicas do subsolo, o gesso favoreceu a produção de cultivar de milho sensível à acidez.
\end{abstract}

Termos de indexação: Acidez subsuperficial, alumínio, cultivar de milho, gesso agrícola, tolerância à acidez.

\footnotetext{
(1) Trabal ho realizado com recursos do Convênio EMBRAPA/PETROFÉRTIL. Parcialmente apresentado no XXIII Congresso Brasileiro de Ciência do Solo, 21 a 27 de julho de 1991, Porto Alegre (RS). Recebido para publicação em setembro de 1995 e aprovado em fevereiro de 1998.

(2) Pesquisador Científico da Seção de Fertilidade do Sol o e Nutrição de Plantas, Instituto Agronômico de Campinas (IAC). Caixa Postal 28, CEP 13001-970 Campinas (SP). Bolsista do CNPq.

(3) Pesquisador Científico da Estação Experimental de Tatuí. CEP 18270-000 Tatuí (SP).
} 


\title{
SUMMARY: EFFECT OF PHOSPHOGYPSUM ON THE YIELD OF CORN VARIETIES WITH DIFFERENTIAL TOLERANCE TO ALUMINUM AT THREE LIMING LEVELS
}

\begin{abstract}
Gypsum is an amendment used to alleviate subsoil acidity. However, there are doubts about its usefor acid soi Is that previously recei ved appli cati ons of limeand fertilizers. Thereare also doubts on the use of gypsum in the cases of the use of high liming rates or for al uminum tolerant crop varieties. This paper describes a limestone and gypsum experiment conducted from 1987 to 1992 at theTatui Experimental Station, Stateof SãoPaulo, Brazil. Theexperiment was on a allic, dark red, clayey latosol and had the objective to evaluate the effect of limestone and gypsum on the production of al umi num tol erant and susceptible corn cultivars, as well as theeffects of thecorrectiveagents on soil acidity. Theexperiment was in a split-spl it-pl ot design, with four replications in randomized blocks. In the main plots 0, 6 and $12 \mathrm{t} \mathrm{ha}^{-1}$ of dolomitic limestonewas applied and in thesubpl ots 0,4 and $8 \mathrm{t} \mathrm{ha}^{-1}$ of phosphogypsum. In thesubsubpl ots, aluminum tolerant and aluminum sensitive corn cultivars were grown. For the four yields obtained (the yield of 1990 was not considered) there was a statistically significant effect of limestone for both types of cultivars. Gypsum had a significant effect only for the aluminum sensitivecultivar and, in this case, the effect was additiveto that of I imestone, theyi el increase being about onehalf of that produced by limestone Liming consi derably affected thesoil reaction in the pl ow layer, increasing the $\mathrm{pH}$ and the contents of $\mathrm{Ca}^{2+}$ and $\mathrm{Mg}^{2+}$ and reducing the buffer extractableacidity, but it did not affect the subsoil, probably because of former applications of limeand sulfur containing fertilizers. Gypsum affected only the $\mathrm{Ca}^{2+}$ and $\mathrm{SO}_{4}{ }^{2-}$ contents of the subsoil, al though in a low degree, consi dering the amounts applied of theamendment, and did not affect soil parameters associated with aci dity. It might becond uded that, even with limited effect on acidity related soil characteristics, gypsum favorably affected the production of Al susceptiblecorn cultivars.
\end{abstract}

Index terms: acid tolerant, aluminum, cultivar, subsoil acidity, phosphogypsum.

\section{INTRODUÇÃO}

O efeito do gesso na redução da acidez do subsolo, promovendo o aprofundamento do sistema radicular, foi demonstrado, pela primeira vez no Brasil, em experimento com milho desenvolvido em Latossolo Vermel ho-Escuroargiloso do Brasil Central, relatado por Ritchey et al. (1980).

O gesso, ou sul fato de cál cio, atua sobre dois fatores que impedem ou dificultam o desenvolvimento radicular das plantas cultivadas: o excesso de al umínio ea deficiência de cálcio. Mesmo em soluç̧̃es nutritivas que contêm alumínio, o sulfato de cálcio tem efeito favorável sobre o crescimento radicular, pela redução do efeito tóxico do alumínio (Adams \& Lund, 1966) ou pela influência favorável do cálcio (Alva et al., 1986; Cameron et al., 1986).

Alguns trabalhos têm revelado o efeito favorável do gesso em culturas anuais. Carvalho et al. (1986) descrevem experimento em que o gesso aumentou a produção de milho, mas não de soja. Na África do Sul, o gesso aumentou a produção de vários cultivos sucessivos de milho (Farina \& Channon, 1988). No estado da Georgia, EUA, foi relatado efeito positivo do gesso sobre culturas de soja, milho, silagem ealfafa (Sumner et al., 1986). Outros casos de efeitos de gesso aplicado em solos ácidos nas produções de culturas são discutidos nas revisões de Raij (1988) e de Shainberg et al . (1989). Trabalho de Raij et al. (1994) mostrou efeito significativo da aplicação do gesso em soja cultivada em Latossol o Roxo distrófico com altos teores de sulfato adsorvido atribuídos a adubações.

O mel horamento vegetal, ao selecionar cultivares tolerantes a alumínio, busca criar genótipos com melhor desenvolvimento radicular em solos ácidos, havendo interesse em avaliar ocomportamento desses materiais genéticos em sol os tratados com gesso para mel horar o ambiente radicular do subsolo.

O objetivo deste trabalho foi avaliar o efeito de gesso, em diferentes níveis de calagem, na produtividade de cultivares de milho com tolerância diferencial a alumínio.

\section{MATERIAL E MÉTODOS}

O experimento foi instalado na Estação Experimental de Tatuí (SP), em um Latossolo Vermel ho-Escuro álico textura argil losa. A análise do solo apresentou os seguintes resultados: P $27 \mathrm{mg} \mathrm{dm}^{-3}$; matéria orgânica $32 \mathrm{~g} \mathrm{dm}^{-3} ; \mathrm{pH} 4,4 \mathrm{em} \mathrm{CaCl}_{2} 0,01 \mathrm{~mol} \mathrm{~L}^{-1}$; em mmol ${ }_{\mathrm{C}} \mathrm{dm}^{-3}$ : K 6,2, Ca 23, Mg 10, H +Al 16 eCTC 112 e V $36 \%$.

O delineamento experimental foi em parcelas subsubdivididas, com quatro repetições, em bl ocos ao acaso. $N$ as parcelas principais, foram aplicadas 0,6 ou 12 t ha-1 de cal cário dol omítico e, nas subparcelas, 
0, 4 e 8 t ha-1 de gesso agrícola. Procurou-se aplicar doses máximas, superiores às normalmente preconizadas, para acentuar o efeito da lixiviação.

Nas subsubparcelas, foram sempre plantados dois cultivares de milho, um tolerante e outro sensível a alumínio. As áreas de cada parcela, subparcela e subsubparcela foram de 300, 84 e $42 \mathrm{~m}^{2}$, respectivamente. $\mathrm{O}$ espaçamento utilizado foi de $1 \mathrm{~m}$ entre as linhas, procurando-se estabelecer uma população de 50.000 plantas por hectare.

As aplicações de calcário dolomítico e de gesso agrícol a foram real izadas em 24 de setembro de 1986. Os corretivos foram pré-incorporados com grade e, posteriormente, incor porados com gradeação dupla a, aproximadamente, 0-25 cm de profundidade.

Cerca de dois meses após a correção do solo, efetuouse o plantio de sorgo, o qual foi descartado em razão da desuniformidade no "stand". Foram feitos, em seguida, cinco plantios anuais de milho, a partir do ano agrícola 1987/88, até o ano agrícola 1991/92. No ano 1989/90, houve problemas no desenvolvimento da cultura, e os dados não foram aproveitados. N os anos em que se colheu o milho, os cultivares plantados, tolerante e sensível, foram, respectivamente, os seguintes: 1987/88 - BR 201 e AG 401; 1988-89 IAC 7974 e IAC 8214; 1990/91 - C 525 e DINA 170; 1991/92 - C 525 e DINA 170.

O plantio de cada ensaio foi sempre realizado em meados de novembro de cada ano agrícola. A adubação básica de plantio constou de $400 \mathrm{~kg} \mathrm{ha-1}$ da fórmula 4-14-8 com $20 \mathrm{~g} \mathrm{~kg}^{-1}$ de Zn. A adubação nitrogenada de cobertura foi feita com $50 \mathrm{~kg}$ ha-1 de $\mathrm{N}$ na forma de uréia, cerca de 35 dias após a emergência do milho.

Foram retiradas amostras compostas de solo dos tratamentos das parcelas experimentais, com doses extremas constituídas de 15 amostras simples, 2, 16 e 41 meses após a aplicação do calcário. As profundidades de amostragem foram variáveis, em camadas de $20 \mathrm{~cm}$, atingindo $60 \mathrm{~cm}$, aos 2 meses, e $80 \mathrm{~cm}$, nas duas amostragens posteriores. As amostras de solo foram preparadas e analisadas segundo Raij \& Quaggio (1983).

Nos cultivos de 1987/88 e de 1991/92, por ocasião do estádio de pendoamento, efetuou-se a amostragem de folhas, retirando, de cada uma de 25 plantas por parcela, o terço central da fol ha da base da espiga. O processamento das amostras e a análise química de macronutrientes e de zinco foram feitos conforme Bataglia et al. (1983).

\section{RESULTADOS E DISCUSSÃO}

$\mathrm{O} \mathrm{pH}$, indicativo da reação do solo, é uma das características que melhor reflete o efeito de um corretivo sobre a acidez do solo, mormente quando a medida éfeita em sol ução sal ina. Assim, os val ores de $\mathrm{pH}$ em $\mathrm{CaCl}_{2}$ 0,01 mol L-1, para três épocas de amostragem e quatro profundidades (Quadro 1), dão uma boa idéia dos efeitos dos dois insumos, calcário e gesso agrícola. Na camada arável, houve efeito pronunciado do calcário, que atingiu efeito máximo aos 16 meses, diminuindo em seguida. 0 gesso não alterou a reação da camada arável, podendo-se atribuir a pequena diminuição do $\mathrm{pH}$ com o tempo à acidificação proporcionada pela aplicação de adubo nitrogenado. J á em profundidade, nem o cal cário nem o gesso influenciaram os valores de $\mathrm{pH}$.

No quadro 2, encontram-se outros resultados de análise de solo, que permitem complementar a discussão, sobre o efeito de cal cário e de gesso sobre o solo. A quantidade máxima de calcário aplicada, de $12 \mathrm{t}$ ha-1, é bem maior do que o val or calculado para $70 \%$ de saturação por bases, que seria de 3,6 t ha-1, considerando um PRNT de 67\%. Mesmo assim, na camada superficial, após 41 meses da aplicação dos corretivos, o valor da saturação por bases estava abaixo de $70 \%$. O calcário promoveu aumento significativo nos teores de cálcio e de magnésio, reduziu os teores de alumínio e até de sulfato, ânion este menos adsorvido ao solo a val ores mais el evados de $\mathrm{pH}$. O potássio não foi afetado, o que pode ser explicado em parte pel os teores el evados do el emento no sol o. Não foi constatado efeito do gesso na camada de solo de 0-20 cm após 41 meses.

Em profundi dade, o que se percebeé, praticamente, a descida de sais solúveis ao longo do perfil, com aumento de cálcio, de magnésio e de sulfato, com efeito maior do gesso do que do cal cário. Percebe-se, também, presença considerável de sulfato adsorvido ao solo, mesmo no tratamento sem gesso, o que, certamente,

\section{Quadro 1. E feito de doses de calcário e de gesso no pH em $\mathrm{CaCl}_{2}$ 0,01 mol L-1 do solo, para diferentes profundidades e épocas de amostragem}

\begin{tabular}{|c|c|c|c|c|c|}
\hline \multirow{2}{*}{$\begin{array}{l}\text { Profundidade } \\
\text { de amostragem }\end{array}$} & \multirow{2}{*}{ Calcário } & \multirow{2}{*}{ Gesso } & \multicolumn{3}{|c|}{ É pocas de amostragem } \\
\hline & & & 2 & 16 & 41 \\
\hline & ---- t ha & -1 & ( & mese & - \\
\hline \multirow[t]{4}{*}{$0-20$} & 0 & 0 & $4,3 b^{(1)}$ & $4,2 b$ & $4,1 b$ \\
\hline & 0 & 8 & $4,3 b$ & $4,1 b$ & $4,1 b$ \\
\hline & 12 & 0 & $5,0 a$ & $5,6 a$ & $5,2 a$ \\
\hline & 12 & 8 & $5,1 a$ & $5,6 a$ & $5,0 a$ \\
\hline \multirow[t]{4}{*}{$20-40$} & 0 & 0 & $4,1 a$ & $4,0 a$ & $4,0 \mathrm{a}$ \\
\hline & 0 & 8 & $4,1 a$ & $4,0 a$ & $4,1 a$ \\
\hline & 12 & 0 & $4,1 a$ & $4,0 a$ & $4,1 a$ \\
\hline & 12 & 8 & $4,1 a$ & $4,0 a$ & $4,1 a$ \\
\hline \multirow[t]{4}{*}{$40-60$} & 0 & 0 & $4,1 a$ & $4,1 a$ & $4,1 a$ \\
\hline & 0 & 8 & $4,1 a$ & $4,1 a$ & $4,2 a$ \\
\hline & 12 & 0 & $4,1 a$ & $4,1 a$ & $4,2 a$ \\
\hline & 12 & 8 & $4,1 a$ & $4,1 a$ & $4,2 a$ \\
\hline \multirow[t]{4}{*}{$60-80$} & 0 & 0 & n.d & $4,1 a$ & 4,1a \\
\hline & 0 & 8 & n.d & $4,1 a$ & $4,1 a$ \\
\hline & 12 & 0 & n.d & $4,1 a$ & $4,2 a$ \\
\hline & 12 & 8 & n.d & $4,2 a$ & $4,2 a$ \\
\hline
\end{tabular}

(1) Valores com as mesmas letras na coluna não diferem entre si pelo teste de Duncan a $5 \%$. 
Quadro 2. Efeito de doses de calcário e de gesso em algumas características químicas(1), para diferentes profundidades do solo de Tatuí, 41 meses após a aplicação dos corretivos

\begin{tabular}{|c|c|c|c|c|c|c|c|c|}
\hline \multicolumn{2}{|c|}{ Tratamento } & \multicolumn{4}{|c|}{ Cátions trocáveis } & \multirow{2}{*}{$\mathrm{SO}_{4}{ }^{2-}$} & \multirow{2}{*}{$\mathbf{v}$} & \multirow{2}{*}{$\mathbf{m}$} \\
\hline Calcário & Gesso & $\mathrm{Ca}^{2+}$ & $\mathrm{Mg}^{2+}$ & $\mathbf{K}^{+}$ & $\mathbf{A l}^{3+}$ & & & \\
\hline \multirow{2}{*}{\multicolumn{2}{|c|}{ - $\mathrm{t} \mathrm{ha}^{-1}$}} & 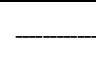 & 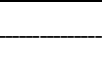 & $\mathrm{mol}_{\mathrm{c}} \mathrm{d}$ & & - & \multirow[t]{2}{*}{ 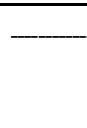 } & $\bar{z}$ \\
\hline & & & & $\operatorname{Pr}$ & dade 0 & $0 \mathrm{~cm}$ & & \\
\hline 0 & 0 & $19 b$ & $6 b$ & $4.5 a$ & $19 a$ & $3,6 a$ & $23 b$ & $39 a$ \\
\hline 0 & 8 & $21 b$ & $5 b$ & $4,0 a$ & $16 a$ & $4,3 a$ & $16 \mathrm{~b}$ & $35 a$ \\
\hline 12 & 0 & $41 a$ & $20 a$ & $3,8 a$ & $2 \mathrm{~b}$ & $1,2 \mathrm{~b}$ & $64 a$ & $3 b$ \\
\hline \multirow[t]{2}{*}{12} & 8 & $41 a$ & $19 a$ & $4,0 a$ & $2 b$ & $1,6 \mathrm{~b}$ & $63 a$ & $3 b$ \\
\hline & & \multicolumn{7}{|c|}{ Profundidade de $20-40 \mathrm{~cm}$} \\
\hline 0 & 0 & $7 a$ & $5 b$ & $3,6 a$ & $24 a$ & $11,4 b$ & $10 a$ & $62 a$ \\
\hline 0 & 8 & $10 a$ & $3 c$ & $2,8 a$ & $25 a$ & $15,5 a$ & $11 a$ & $60 a$ \\
\hline 12 & 0 & $8 a$ & $7 a$ & $2,6 a$ & $22 a$ & $12,9 a b$ & $13 a$ & $56 a$ \\
\hline \multirow[t]{2}{*}{12} & 8 & $8 a$ & $6 a$ & $2,4 a$ & $23 a$ & $15,1 a$ & $10 a$ & $59 a$ \\
\hline & & \multicolumn{7}{|c|}{ Profundidade de $40-60 \mathrm{~cm}$} \\
\hline 0 & 0 & $10 \mathrm{~b}$ & $4 c$ & $2,2 a$ & $22 a$ & $11,0 \mathrm{~b}$ & $12 a$ & $58 a$ \\
\hline 0 & 8 & $13 a b$ & $5 b c$ & $1,6 a$ & $19 a b$ & $17,2 a$ & $14 a$ & $49 a b$ \\
\hline 12 & 0 & $11 a b$ & $7 a b$ & $2,0 a$ & $19 a b$ & $12,9 \mathrm{~b}$ & $15 a$ & $50 a b$ \\
\hline \multirow[t]{2}{*}{12} & 8 & $14 a$ & $8 a$ & $2,1 a$ & $17 b$ & $16,0 a$ & $18 a$ & $41 b$ \\
\hline & & \multicolumn{7}{|c|}{ Profundidade de $60-80 \mathrm{~cm}$} \\
\hline 0 & 0 & $6 b$ & $3 b$ & $1,7 a$ & $21 a b$ & $6,2 b$ & $8 a$ & $61 a$ \\
\hline 0 & 8 & $10 a$ & $5 a b$ & $1,3 a$ & $18 \mathrm{~b}$ & $14,1 a$ & $13 a$ & $52 a$ \\
\hline 12 & 0 & $7 b$ & $5 a b$ & $1,4 a$ & $22 a b$ & $6,5 b$ & $11 a$ & $62 a$ \\
\hline 12 & 8 & $11 a$ & $7 a$ & $1,6 a$ & $23 a$ & $15,9 a$ & $14 a$ & $54 a$ \\
\hline
\end{tabular}

${ }^{(1)}$ Valores com as mesmas letras na coluna não diferem entre si pelo teste de Duncan a 5\%.

decorre de adubações anteriores com fertilizantes que contêm enxofre, como sulfato de amôni o e superfosfato simples. Como conseqüência, os efeitos do gesso sobre os teores de sulfato são mais acentuados nas camadas mais profundas, de 40-60 e 60-80 cm de profundidadee quiçá nas maiores profundidades, que não foram amostradas. A quantidademáxima aplicada, de8t ha-1, promoveu alterações modestas no perfil do solo, comparadas com os resultados mostrados por Souza \& Ritchey (1986) para a região dos Cerrados, utilizando quantidades próximas de gesso.

Os resultados deste experimento diferem, substancial mente, dos obtidos por Ritchey et al. (1980), em LatossoloVermelho-E scuroargiloso, queverificaram efeito considerável do gesso em reduzir efetivamente a acidez do subsolo. A diferença de resultados pode ser explicada por duas causas principais. A primeira diz respeito à atividade da argila dos dois solos, tendo o solo do trabalho de Ritchey et al. (1980) argila de baixa atividade, o que é refletido na CTC e nos teores de alumínio trocável baixos, enquanto no solo deste trabal ho a atividade da argila é considerável e, o que é mais marcante, a CTC aumenta em profundidade, atingindo $160 \mathrm{mmol}_{\mathrm{c}} \mathrm{dm}^{-3}$ na camada de 60 a $80 \mathrm{~cm}$ de profundidade, além de o solo apresentar teores bastante altos de alumínio. A segunda causa é o acúmulo considerável de sulfato no perfil do solo deste trabalho, enquanto o solo de Ritchey et al. (1980) era virgem e praticamente desprovido de bases trocáveis. I sso serve também para explicar porque o calcário pouco influiu no subsolo neste trabalho, ao contrário do observado por Quaggio et al . (1982) em um L atossolo Roxo de Cerrado; por Quaggio et al. (1985), em um Cambissolo, e por Souza \& Ritchey (1986), em um Latossolo Vermelho-E scuro argil oso de cerrado, casos em que houve efeito considerável da calagem na correção da acidez do subsolo. Neste caso, como o solo já continha quantidades consideráveis de sulfato, cál cio e magnésio adsorvidos, os íons devem ter sido lixiviados, em sua maior parte, para camadas mais profundas do que as amostradas.

Também não foram identificadas perdas consideráveis de $\mathrm{Mg}$ e de $\mathrm{K}$, ao menos de um tratamento em relação a outro, assunto que merece al guns comentários. Muitas vezes, a redução de teores desses cátions na camada arável é considerada como perda, o que nem sempre é o caso. Somente com a lixiviação de sulfato para fora do alcance das raízes, poderão ocorrer perdas efetivas de bases, quelixiviarão com o ânion. Com aplicações de cal cário dol omítico, as 
perdas de magnésio, proporcionadas pela aplicação de gesso, não devem preocupar. O solo deste trabal ho é bastante rico em potássio (Quadro 2), e a reciclagem via biomassa devolve o elemento à superfície, mantendo os teores el evados na superfície.

Os teores de sulfato contidos nas diferentes camadas do solo, apresentados no quadro 2, são representativos do comportamento desse ânion, que é fortemente adsorvido em sol os que contêm teores elevados deóxidos hidratados de ferroealumínio, como os do presente caso (Chao et al., 1965; Raij \& Peech, 1972). O sulfatoé mais fortementeretido em sol os mais ácidos e com menor carga elétrica negativa (Raij \& Peech, 1972; Camargo \& Raij, 1989). I sso e a repul são de sulfato por fosfato, ânion com adsorção preferencial na camada arável, explicam os teores mais baixos de $\mathrm{SO}_{4}{ }^{2-}$ na superfície do solo. Nas camadas mais profundas, nota-se o acúmulo de sulfato, em parte já existentenosolo, nos tratamentos sem gesso, acrescidos por adições feitas no presente ensaio, nos tratamentos com gesso. $\mathrm{O}$ aumento de $\mathrm{SO}_{4}{ }^{2-}$ é mais evidente na camada de 60 a $80 \mathrm{~cm}$ de profundidade, indicando que o efeito deveter-seestendidotambém em profundidades ainda maiores do solo. Dequal quer forma, fica evidente que o íon $\mathrm{SO}_{4}{ }^{2-}$ é fortemente adsorvido no subsolo, aí permanecendo por muitos anos, informação das mais importantes para a diagnose da disponibilidade de enxofre em solos.

No quadro 3, são apresentados os efeitos médios, de cal cário e de gesso, nas produções de milho para os dois cultivares. O cultivar tolerante a alumínio respondeu significativamente à aplicação de cal cário em todos os anos, com um efeito médio, nos quatro anos, de $26 \%$ de aumento de produção, enquanto o gesso não teve efeito significativo, proporcionando aumento médio de produção de apenas $4 \%$. Já o cultivar sensível apresentou também resposta significativa à aplicação de calcário em todas as alternativas apresentadas no quadro 3 , com aumento médio de produção de $29 \%$. O efeito do gesso foi significativo em apenas dois dos quatro anos eno efeito médio, que representou um aumento de produção de $12 \%$. Por questões de disponibilidade de sementes, foi necessário mudar os cultivares todos os anos e fica difícil saber se, nos casos em que não houve significância do efeito do gesso, isso se deveu a diferenças genéticas do milho ou simplesmente a um maior erro experimental.

Os resultados de análises químicas realizadas em amostras de fol has coletadas em 1988 (Quadro 4) e 1992 (Quadro 5) revelaram grandes efeitos do cal cário nos teores de cálcio e magnésio, embora os teores desses nutrientes na camada arável sejam considerados suficientes (Quadro 2). O tratamento com gesso afetou os teores de $\mathrm{K}, \mathrm{Ca}$ e $\mathrm{Mg}$, porém de maneira não-consistente, ou seja, no ano de 1988, observou-se o efeito apenas no $\mathrm{Ca}$, enquanto, no ano de 1992, os efeitos foram para K e Mg. Efeitos mais generalizados nos teores dos nutrientes foram observados para cultivares, notando-se repetitivas as diferenças varietais para K, Ca e S. Os cultivares sensíveis (AG 401 e D 170) apresentaram menores

Quadro 3. Produções médias de dois cultivares de milho, tolerante e sensível a alumínio, para doses aplicadas de calcário (C) e de gesso (G)

\begin{tabular}{|c|c|c|c|c|c|}
\hline \multirow[t]{2}{*}{ Tratamento } & \multicolumn{5}{|c|}{ Produção de milho para os anos agrícolas ${ }^{(1)}$} \\
\hline & $1987 / 88$ & $1988 / 89$ & 1990/91 & 1991/92 & Média \\
\hline & \multicolumn{5}{|c|}{$-\mathrm{kg} \mathrm{ha}^{-1}$} \\
\hline & \multicolumn{5}{|c|}{ Cultivar tolerante a alumínio } \\
\hline $\mathrm{C}_{0}$ & $5.786 b$ & $4.135 b$ & $3.773 \mathrm{~b}$ & $3.670 b$ & $4.341 c$ \\
\hline $\mathrm{C}_{6}$ & $6.253 b$ & $4.801 a$ & $3.924 b$ & $4.580 a$ & $4.890 \mathrm{~b}$ \\
\hline $\mathrm{C}_{12}$ & $7.577 a$ & $5.117 a$ & $4.424 a$ & $4.780 a$ & $5.474 a$ \\
\hline $\mathrm{G}_{0}$ & $6.476 a$ & $4.477 a$ & $3.844 a$ & $4.217 a$ & $4.785 a$ \\
\hline $\mathrm{G}_{4}$ & $6.533 a$ & $4.730 a$ & $4.123 a$ & $4.283 a$ & $4.940 \mathrm{a}$ \\
\hline \multirow[t]{2}{*}{$\mathrm{G}_{8}$} & $6.607 a$ & $4.849 a$ & $4.153 a$ & $4.530 a$ & $4.979 a$ \\
\hline & \multicolumn{5}{|c|}{ Cultivar tolerante a alumínio } \\
\hline $\mathrm{C}_{0}$ & $6.042 b$ & $3.865 b$ & $4.088 \mathrm{~b}$ & $4.104 b$ & $4.525 c$ \\
\hline $\mathrm{C}_{6}$ & $6.512 b$ & $4.944 a$ & $5.307 a$ & $5.121 a$ & $5.489 \mathrm{~b}$ \\
\hline $\mathrm{C}_{12}$ & $7.410 \mathrm{a}$ & $5.016 a$ & $5.376 a$ & $5.607 a$ & $5.835 a$ \\
\hline $\mathrm{G}_{0}$ & $6.134 b$ & $4.340 a$ & $4.733 a$ & $4.532 b$ & $4.935 b$ \\
\hline $\mathrm{G}_{4}$ & $6.803 a b$ & $4.656 a$ & $4.995 a$ & $5.101 a$ & $5.400 a$ \\
\hline $\mathrm{G}_{8}^{4}$ & $7.027 a$ & $4.828 a$ & $5.024 a$ & 5.199a & $5.512 a$ \\
\hline
\end{tabular}

\footnotetext{
(1) Produções com a mesma letra, em cada coluna, não diferem entre si pelo teste de Duncan a 5\%.
} 
Quadro 4. Teores de macronutrientes e de zinco em amostras de folhas da base da espiga de cultivares de milho em função de tratamentos extremos de calagem (C) e de gesso (G), para o ano agrícola 1987/88, e conclusões da análise de variância

\begin{tabular}{|c|c|c|c|c|c|c|c|c|}
\hline Tratamento & Cultivar & $\mathbf{N}$ & $\mathbf{0}$ & $\mathbf{K}$ & $\mathbf{C a}$ & Mg & $\mathbf{S}$ & $\mathbf{Z n}$ \\
\hline & & & & - & & & - & $\mathrm{mg} \mathrm{kg}^{-1}$ \\
\hline \multirow{2}{*}{$\mathrm{C}_{0} \mathrm{G}_{0}$} & BR 201 & 29,2 & 2,5 & 24,0 & 3,7 & 1,2 & 1,9 & 19,1 \\
\hline & AG 401 & 29,2 & 2,5 & 22,9 & 3,9 & 1,2 & 2,5 & 19,1 \\
\hline \multirow{2}{*}{$\mathrm{C}_{0} \mathrm{G}_{8}$} & BR 201 & 29,7 & 2,4 & 25,4 & 4,1 & 1,2 & 2,0 & 21,5 \\
\hline & AG 401 & 29,6 & 2,5 & 21,2 & 4,4 & 1,3 & 2,4 & 21,1 \\
\hline \multirow[t]{2}{*}{$\mathrm{C}_{12} \mathrm{G}_{0}$} & BR 201 & 26,9 & 2,5 & 22,9 & 4,5 & 2,2 & 1,7 & 19,3 \\
\hline & AG 401 & 31,4 & 2,7 & 22,3 & 4,5 & 2,4 & 2,2 & 19,0 \\
\hline \multirow{2}{*}{$\mathrm{C}_{12} \mathrm{G}_{8}$} & BR 201 & 27,0 & 2,6 & 24,4 & 4,4 & 2,1 & 1,7 & 19,9 \\
\hline & AG 401 & 31,7 & 2,7 & 19,7 & 4,9 & 2,1 & 2,4 & 19,9 \\
\hline \multicolumn{9}{|l|}{ Teste $\mathbf{F}^{(1)}$} \\
\hline \multicolumn{2}{|l|}{ Calcário } & ns & ns & ns & $* *$ & $* *$ & ns & ns \\
\hline \multicolumn{2}{|l|}{ Gesso } & ns & ns & ns & $*$ & ns & ns & ns \\
\hline \multicolumn{2}{|l|}{ Cal cário x gesso } & ns & ns & ns & ns & ns & ns & $* *$ \\
\hline \multicolumn{2}{|l|}{ Cultivar } & $*$ & ns & $*$ & $*$ & $*$ & $* *$ & ns \\
\hline \multicolumn{2}{|l|}{ Cal cário x cultivar } & ns & ns & ns & ns & ns & ns & ns \\
\hline \multicolumn{2}{|l|}{ Gesso $\times$ cultivar } & ns & ns & ns & ns & ns & ns & ns \\
\hline \multicolumn{2}{|l|}{ Cultivar x cal cário x gesso } & ns & ns & $*$ & ns & ns & ns & ns \\
\hline
\end{tabular}

(1) São indicadas diferenças não-significativas (ns) e significativas a 5\% (*) e 1\% (**).

Quadro 5. Teores de macronutrientes e de zinco em amostras de folhas da base da espiga de cultivares de milho em função de tratamentos extremos de calagem (C) e de gesso (G), para o ano agrícola 1991/92, e conclusões da análise de variância

\begin{tabular}{|c|c|c|c|c|c|c|c|c|}
\hline Tratamento & Cultivar & $\mathbf{N}$ & $\mathbf{0}$ & $\mathbf{K}$ & $\mathrm{Ca}$ & Mg & $\mathbf{s}$ & Zn \\
\hline & & & & 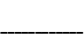 & 1 & & - & $\mathrm{mg} \mathrm{kg}^{-1}$ \\
\hline \multirow[t]{2}{*}{$\mathrm{C}_{0} \mathrm{G}_{0}$} & C 525 & 22,5 & 1,9 & 23,2 & 4,2 & 1,7 & 2,3 & 16,0 \\
\hline & D 170 & 21,6 & 1,8 & 20,7 & 4,1 & 1,6 & 1,8 & 21,3 \\
\hline \multirow{2}{*}{$\mathrm{C}_{0} \mathrm{G}_{8}$} & C 525 & 20,7 & 1,7 & 22,0 & 4,3 & 1,6 & 2,0 & 16,8 \\
\hline & D 170 & 22,5 & 2,0 & 18,8 & 5,0 & 1,5 & 1,8 & 25,0 \\
\hline \multirow[t]{2}{*}{$\mathrm{C}_{12} \mathrm{G}_{0}$} & C 525 & 19,7 & 1,9 & 20,8 & 4,8 & 2,8 & 1,9 & 14,5 \\
\hline & D 170 & 17,8 & 1,6 & 17,0 & 5,5 & 3,0 & 1,6 & 17,8 \\
\hline \multirow[t]{2}{*}{$\mathrm{C}_{12} \mathrm{G}_{8}$} & C 525 & 22,2 & 1,8 & 19,7 & 4,6 & 2,7 & 2,2 & 16,0 \\
\hline & D 170 & 19,6 & 1,8 & 16,9 & 5,2 & 2,7 & 1,7 & 19,3 \\
\hline \multicolumn{9}{|l|}{ Teste $F^{(1)}$} \\
\hline \multicolumn{2}{|l|}{ Calcário } & ns & ns & $*$ & $* *$ & $* *$ & ns & ns \\
\hline \multicolumn{2}{|l|}{ Gesso } & ns & ns & $*$ & ns & $*$ & ns & ns \\
\hline \multicolumn{2}{|l|}{ Calcário x gesso } & ns & ns & ns & ns & ns & ns & ns \\
\hline \multicolumn{2}{|l|}{ Cultivar } & ns & ns & $* *$ & $* *$ & ns & $* *$ & $* *$ \\
\hline \multicolumn{2}{|l|}{ Calcário x cultivar } & ns & ns & ns & ns & ns & ns & $*$ \\
\hline \multicolumn{2}{|l|}{ Gesso x cultivar } & ns & $*$ & ns & $*$ & ns & ns & ns \\
\hline \multicolumn{2}{|l|}{ Calcário x gesso x cultivar } & ns & ns & ns & ns & ns & ns & ns \\
\hline
\end{tabular}

\footnotetext{
(1) São indicadas diferenças não-significativas (ns) e significativas a $5 \%(*)$ e $1 \%$ (**).
} 
teores deK emaiores de Ca, sem, contudo, mostrarem o mesmo tipo de tendência com relação ao enxofre. Os efeitos de interações entre os tratamentos nos teores de nutrientes não apresentaram resultados conclusivos. Dessa forma, observaram-se efeitos da interação cal cário x gesso nos teores de Zn apenas no ano de 1988; da interação cal cário x cultivar nos teores de Zn apenas em 1992; da interação gesso $x$ cultivar nos teores de K e Ca em 1992; e da interação calcário x gesso apenas no teor de $\mathrm{K}$ em folhas coletadas em 1988. Com a baixa dose de nitrogênio aplicada, esperava-se que os corretivos pudessem, estimular a absorção de nitrogênio do subsolo, mas tal efeito não foi observado.

No quadro 6, são apresentados os efeitos médios das doses aplicadas de gesso, dentro de cada dose de calcário e para cada tipo de cultivar. Houve significância para o efeito do gesso apenas para as duas doses mais baixas decal cário do cultivar sensível a alumínio. Parece bastante claro, pela mesma ordem de magnitude das respostas a gesso, dentro de qualquer uma das doses de calcário, que os dois insumos têm efeitos independentes sobre a produção.

A ausência de efeito do gesso nos parâmetros de acidez, que foram medidos eapresentados nos quadros 1 e 2, não elimina a possibilidade de o insumo ter atuado na redução dos efeitos nocivos do alumínio sobre o desenvol vimento radicular. I sso porque, mesmo em solução, o sulfato de cál cio tem esse efeito (Adams \& Lund, 1966). Assim, é possível que o aumento de sulfato nas camadas mais profundas do solo, ricas em alumínio (Quadro 2), tenha proporcionado condições mais favoráveis ao desenvolvimento radicular. Isso

\section{Quadro 6. Produções médias de quatro anos de dois cultivares de milho, tolerante e sensível a alumínio, para doses de gesso (G) em presença de três doses de calcário (C)}

\begin{tabular}{|c|c|c|c|}
\hline \multicolumn{2}{|c|}{ Tratamento } & \multicolumn{2}{|c|}{ Produção de milho para ${ }^{(1)}$} \\
\hline & & \multicolumn{2}{|c|}{ Cultivar tolerante Cultivar sensível } \\
\hline & & - $\mathrm{kgh}$ & $a^{-1}$ \\
\hline \multirow{3}{*}{$\mathrm{C}_{0}$} & $\mathrm{G}_{0}$ & $4.300 a$ & $4.229 \mathrm{~b}$ \\
\hline & $\mathrm{G}_{4}$ & $4.360 a$ & $4.552 a b$ \\
\hline & $\mathrm{G}_{8}$ & $4.362 a$ & 4.793a \\
\hline \multirow[t]{3}{*}{$\mathrm{C}_{6}$} & $\mathrm{G}_{0}$ & $4.663 a$ & $5.041 b$ \\
\hline & $\mathrm{G}_{4}$ & $4.943 a$ & $5.619 a$ \\
\hline & $\mathrm{G}_{8}$ & $5.063 a$ & $5.817 a$ \\
\hline \multirow[t]{3}{*}{$C_{12}$} & $\mathrm{G}_{0}$ & $5.331 a$ & $5.536 a$ \\
\hline & $\mathrm{G}_{4}$ & $5.398 a$ & $5.927 a$ \\
\hline & $\mathrm{G}_{8}$ & $5.695 a$ & $6.041 a$ \\
\hline
\end{tabular}

(1) Produções de cada cultivar seguidas da mesma letra não diferem entre si pelo teste de Duncan a $5 \%$. estaria coerente, inclusive, com os efeitos independentes dos dois insumos, o cal cário atuando mais na melhoria da camada superficial do solo, no presente ensaio, ao passo que o gesso teria uma ação mais em profundidade. Também por isso o efeito do calcário é mais importante do que do gesso, pois, por mais importância que se queira atribuir ao desenvolvimento radicular no subsolo, as raízes na camada arável terão semprea mai or contribuição para a produtividade.

\section{CONCLUSÕES}

1. O calcário corrigiu a acidez apenas da camada arável do solo, enquanto o gesso, embora sem alterar parâmetros de acidez do solo, influiu nas camadas mais profundas do solo.

2. O calcário influiu significativamente nas produções de mil ho dos cultivares tolerantee sensível a alumínio, e o gesso apenas na do último.

3. O aumento de produção de milho do cultivar sensível à acidez proporcionado pelo gesso foi independente do efeito do cal cário, de cerca da metade em magnitude.

\section{LITERATURA CITADA}

ADAMS, F. \& LUND, Z.F. Effect of chemical activity of soil solution aluminum on cotton root penetration of acid subsoils. Soil Sci., 101:193-198, 1966.

ALVA, A.K.; EDWARDS, D.G.; ASHER, C.J . \& BLAMEY, F.P.C. Effects of phosphorus/aluminum molar ratio and calcium concentration on plant response to aluminum toxicity. Soil Sci. Soc. Am. J., 50:133-137, 1986.

BATAGLIA, O.C.; FURLANI, A.M.C.;TEIXEIRA,J .P.F.; FURLANI, P.R. \& GALLO, J.R. Métodos de análise química de plantas. Campinas, InstitutoAgronômico, 1983. 48p. (Bol etimTécnico, 78)

CAMARGO, O.A. \& RAIJ , B. van. Movimento de gesso em amostras de latossol os com diferentes propriedades el etroquímicas. R. Bras. Ci. Solo, 13:275-280, 1989.

CAMERON, R.S.; RITCHIE, G.S.P. \& ROBSON. Relative toxicities of inorganic aluminum complexes to barley. Soil Sci. Soc. Am. J., 50:1231-1236, 1986.

CARVALHO, L.J.C.B.; GOMIDE, R.L.; RODRIGUES, G.C.; SOUZA, D.M.G. \& FREITAS J r., E. Resposta do milho à aplicação de gesso e déficit hídrico em solos de cerrado. In: SEMINÁRIO SOBRE O USO DE FOSFOGESSO NA AGRICULTURA, Brasília, 1986. Anais. Brasília, EMBRAPA-DDT, 1986. p.61-83.

CHAO, T.T.; HARWARD, M.E. \& FANG, S.C. Exchange reactions between hydroxyl and sulfate ions by soils. Soil Sci., 99:104108, 1965.

FARINA, M.P.W. \& CHANNON, P. Acid-subsoil amelioration. II. Gypsum effects on growth and subsoil chemical properties. Soil Sci. Soc. Am. J., 52:175-180, 1988. 
QUAGGIO, J.A.; MASCARENHAS, H.A.A. \& BATAGLIA, O.C. Respostas da soja à aplicação de doses crescentes de cal cário em Latossolo Roxo distrófico de cerrado. II Efeito residual. R. Bras. Ci. Solo, 6:113-118, 1982.

QUAGGIO, J .A.; RAMOS, V.J .; BATAGLIA, O.C.; RAIJ , B. van \& SAKAI, M. Calagem para a sucessão batata-triticale-milho usando cal cário com diferentes teores de magnésio. Bragantia, 44:391-406, 1985.

RAIJ, B. van. Gesso agrícola na melhoria do ambiente radicular no subsolo. São Paulo, Associação Nacional para Difusão de Adubos e Corretivos Agrícolas, 1988. 88p.

RAIJ, B. van; MASCARENHAS, H.A.A.; PEREIRA, J.C.V.N.A.; IGUE, T. \& SORDI, G. Efeito de calcário e de gesso para soja cultivada em latossolo roxo ácido saturado com sulfato. R. Bras. Ci. Solo, 18:305-312, 1994.

RAIJ, B. van \& QUAGGIO, J.A. Métodos de análise de solo para fins defertilidade. Campinas, I nstitutoAgronômico, 1983. 31p. (Boletim Técnico, 81)
RAIJ, B. van \& PEECH, M. Electrochemical properties of some Oxisols and Alfisols of the tropics. Soil Sci. Soc. Am. Proc., 36:587-593, 1972.

RITCHEY, K.D.; SOUZA, D.M.G.; LOBATO, E. \& CORREA, O. Calcium leaching to increase rooting depth in a Brazilian savannah Oxisol. Agron. J., 72:40-44, 1980.

SHAINBERG, I.; SUMNER, M.E.; MILLER, W.P;FARINA, M.P.W. \& FEY, M.V. Use of gypsum on soils: a review. Adv. Soil Sci., 9:1-111, 1989.

SOUZA, D.M.G. \& RITCHEY, K.D. Uso de gesso no sol o de cerrado. In: SEMINÁRIO SOBRE O USO DE FOSFOGESSO NA AGRICULTURA, 1., Brasília, 1986. Anais. Brasília, EMBRAPA-DDT, 1986. p.119-144.

SUMNER, M.E.; SHAHANDEH, H.; BOUTON, J \& \& HAMMEL, J . Amelioration of an acid soil profile through deep liming and surface application of gypsum. Soil Sci. Soc. Am. J ., 50:12541278, 1986. 